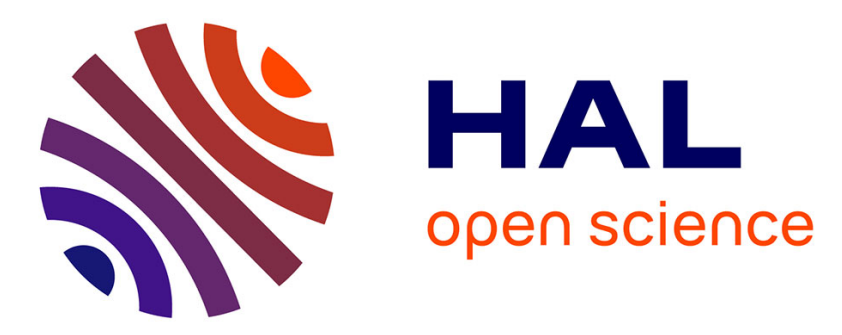

\title{
On the existence of hysteresis in a transition to chaos after a single bifurcation
}

C. Tresser, P. Coullet, A. Arneodo

\section{To cite this version:}

C. Tresser, P. Coullet, A. Arneodo. On the existence of hysteresis in a transition to chaos after a single bifurcation. Journal de Physique Lettres, 1980, 41 (10), pp.243-246. 10.1051/jphyslet:019800041010024300 . jpa-00231770

\section{HAL Id: jpa-00231770 https://hal.science/jpa-00231770}

Submitted on 1 Jan 1980

HAL is a multi-disciplinary open access archive for the deposit and dissemination of scientific research documents, whether they are published or not. The documents may come from teaching and research institutions in France or abroad, or from public or private research centers.
L'archive ouverte pluridisciplinaire HAL, est destinée au dépôt et à la diffusion de documents scientifiques de niveau recherche, publiés ou non, émanant des établissements d'enseignement et de recherche français ou étrangers, des laboratoires publics ou privés. 


\title{
On the existence of hysteresis in a transition to chaos after a single bifurcation
}

\author{
C. Tresser, P. Coullet \\ Equipe de Mécanique Statistique $\left({ }^{*}\right)$, Université de Nice, \\ Parc Valrose, 06034 Nice Cedex, France \\ and A. Arneodo \\ Laboratoire de Physique Théorique (**), Université de Nice, Parc Valrose, 06034 Nice Cedex, France
}

(Reçu le 20 décembre 1979, accepté le 27 mars 1980)

\begin{abstract}
Résumé. - Nous étudions et classifions des transitions discontinues vers des comportements chaotiques survenant après une seule bifurcation dans des endomorphismes de $\mathbb{R}$. Cette classification peut être étendue à des systèmes dynamiques plus réalistes. Elle repose sur l'existence possible du mécanisme d'hysteresis. Nous discutons de l'intermittence comme un test de non-existence d'hysteresis.
\end{abstract}

\begin{abstract}
We study and classify discontinuous transitions to chaotic behaviour occurring after a single bifurcation in real endomorphisms. This classification which can be extended to more realistic dynamical systems, relies on the possible existence of the hysteresis mechanism. We discuss the intermittency phenomenon as an evidence for transitions without hysteresis.
\end{abstract}

1. Introduction. - These last years, many efforts have been devoted to understand the transition to turbulent behaviour. These efforts have been stimulated by Lorenz's numerical investigations [1] of a non-periodic deterministic flow and a paper of Ruelle and Takens [2] on the nature of turbulence where these authors proved the existence of structurally stable strange Axiome $A$ attractors in the neighbourhood of some quasiperiodic flows.

We can describe the transition to stochasticity in dynamical systems as continuous or discontinuous according as it occurs respectively after an accumulation of bifurcations [3, 4], or after a finite number of apparent bifurcations [1]. Renormalization group methods have been used in order to describe universal aspects of continuous transitions for simple dynamical systems [5-9]. In analogy with phase transitions, we do not expect any universal property when the transition is discontinuous; we generally observe a hysteresis phenomenon as e.g. in Lorenz model [10]. Abrupt transitions to turbulence with hysteresis have actually been experimentally exhibited by Maurer and Libchaber [11] on Bénard convection

(*) Laboratoire de Physique de la Matière Condensée Associé au C.N.R.S.

(**) Equipe de Recherche Associée au C.N.R.S. in liquid helium. The same results have been obtained by Gollub and Benson in a similar experiment on water [12]. However, for a large number of dynamical systems (endomorphisms of an interval, diffeomorphisms of $\mathbb{R}^{2}$, differential equations), hysteresis has never been numerically displayed although the stochastic transition seems to be discontinuous [13]. Yet in this case we observe intermittency, as described by Pomeau and Manneville in [14].

The aim of this letter is to study the existence of hysteresis and related phenomena for discontinuous transitions to chaos. We mainly restrict ourselves to non-invertible one-dimensional difference equations where exact results can be derived; other numerical results on flows and diffeomorphisms will be reported elsewhere [13].

Let us start quoting a theorem which may be deduced from lemma 3.1 and proposition 2.1 in the reference [16] by Misiurewicz.

Let $\mathrm{F}$ be the class of $C^{3}$ functions $f:[0,1] \rightarrow \mathbb{R}$, such that $f$ has a negative Schwarzian derivative [15] $\left({ }^{1}\right)$, and only one critical point $\left.\bar{x} \in\right] 0,1[$, and satisfies $f(0)=f(1)$. Then :

( ${ }^{1}$ ) The Schwarzian derivative of a $C^{3}$ map is defined by :

$$
S f=\frac{f^{\prime \prime \prime}}{f^{\prime}}-\frac{3}{2}\left(\frac{f^{\prime \prime}}{f^{\prime}}\right)^{2} .
$$


for any $y>0$, if $\mathcal{F}:[-y, 1+y] \rightarrow \mathbb{R}$ is such that $\left.\mathcal{F}\right|_{[0,1]}=f$ is in $\mathrm{F}$ and satisfies :

$$
f(\bar{x})>1, f^{\prime}(0)=\alpha>1, f^{\prime}(1)=\beta<0,
$$

then, for $\mu$-almost all $x \in[0,1]$, some iterate of $x$ under $\mathcal{F}$ is not in $[0,1]$ (where $\mu$ stands for Lebesgue measure).

Now let $f_{0}: \mathbb{R} \rightarrow \mathbb{R}$ be a $C^{3}$ function with negative Schwarzian derivative such that (Fig. 1)

$$
\begin{array}{ll}
f_{0}^{\prime}(x)<0 & \text { if } \quad x<\bar{X}_{1} \\
f_{0}^{\prime}(x)>0 & \text { if } \quad \bar{X}_{1}<x<\bar{X}_{2} \\
f_{0}^{\prime}(x)<0 & \text { if } x>\bar{X}_{2} .
\end{array}
$$

In order to study transitions to stochasticity we consider families $f_{\lambda}=f_{0}+\lambda$ which present a discontinuous transition for $\lambda=0$.

For $\lambda \leqslant 0$, we define : $f_{\lambda}$,

- $a_{\lambda} \leqslant b_{\lambda}<X_{\lambda}^{*}$ as the fixed points of the function

- $c_{\lambda}$ and $\tilde{X}_{\lambda}$ respectively as the smallest and greatest values of $x$ such that $f_{\lambda}(x)=b_{\lambda}$.

Some conditions on $f_{0}$ satisfied in $C^{3}$ open sets, determine whether the transition occurs with or without hysteresis when $\lambda$ crosses zero. We suppose that for $\lambda<0$, the fixed point $a_{\lambda}$ is stable on the whole range of parameters we explore. With this class of models we can also describe cases where the hysteresis is incomplete, i.e. cases where decreasing adiabatically $\lambda$ from values slightly greater than zero, we cannot trace back the history of bifurcations to the saddle node bifurcation which gives rise to the fixed point $X_{i}^{*}$. The general diagram corresponding to these situations is represented on figure 2. Keeping in mind that we are studying transitions to chaos, we would have to require positive Kolmogorov entropy for the attractor ( $A$ on figure 2 ) we arrive when the fixed point $a_{\lambda}$ disappears. Since it might be impossible to satisfy this condition on a set of parameters with positive measure when $f$ is at least

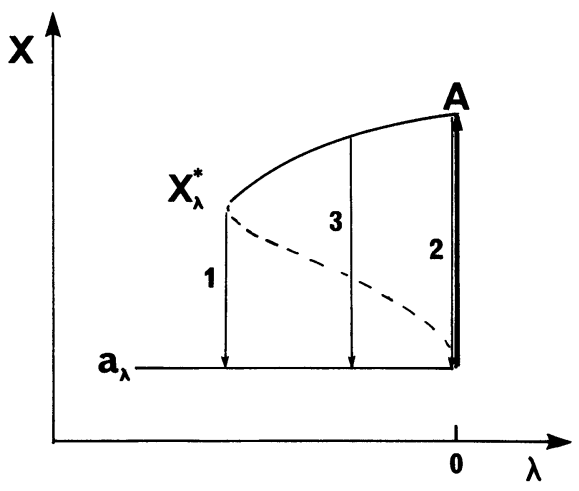

Fig. 2. - General bifurcation diagram associated to a discontinuous transition : 1) with complete hysteresis; 2) without hysteresis; 3) with incomplete hysteresis.

$C^{1}$ [16], we shall be content with cases with positive topological entropy.

We now proceed to classify the various situations that may occur.

CASE 1. - Transition with complete hysteresis.

This is realized by imposing

$$
f_{\lambda}\left(\bar{X}_{2}\right)<\tilde{X}_{\lambda} \text { for } \lambda \leqslant 0 .
$$

Hence for $\lambda<0$ we have $f_{\lambda}^{2}\left(\bar{X}_{2}\right)>b_{\lambda}$ and the invariant interval $\left[f_{\lambda}^{2}\left(\bar{X}_{2}\right), f_{\lambda}\left(\bar{X}_{2}\right)\right]$ contains the attractor $A_{\lambda}$ of the endomorphism $\left.f_{\lambda}\right|_{\left[b_{\lambda}, \tilde{X}_{\lambda]}\right.}$ (Fig. 3). As a consequence $A_{\lambda}$ is bounded away from the fixed point $b_{\lambda}$ and we do trace back the $A_{\lambda}$ history when the parameter $\lambda$ is decreased adiabatically.

CASE 2. - Transition without hysteresis.

This is guaranted by the set of conditions (Fig. 3) :

$$
\begin{aligned}
& f_{0}\left(\bar{X}_{2}\right)>\tilde{X}_{0} \\
& f_{0}^{2}\left(\bar{X}_{2}\right) \geqslant c_{0} .
\end{aligned}
$$

Applying the above mentioned theorem (Section 2), the first condition implies that almost every initial condition in $\left[a_{0}, \tilde{X}_{0}\right]$ has an iterate under $f_{0}$ out of this interval. On the other hand, the second condition

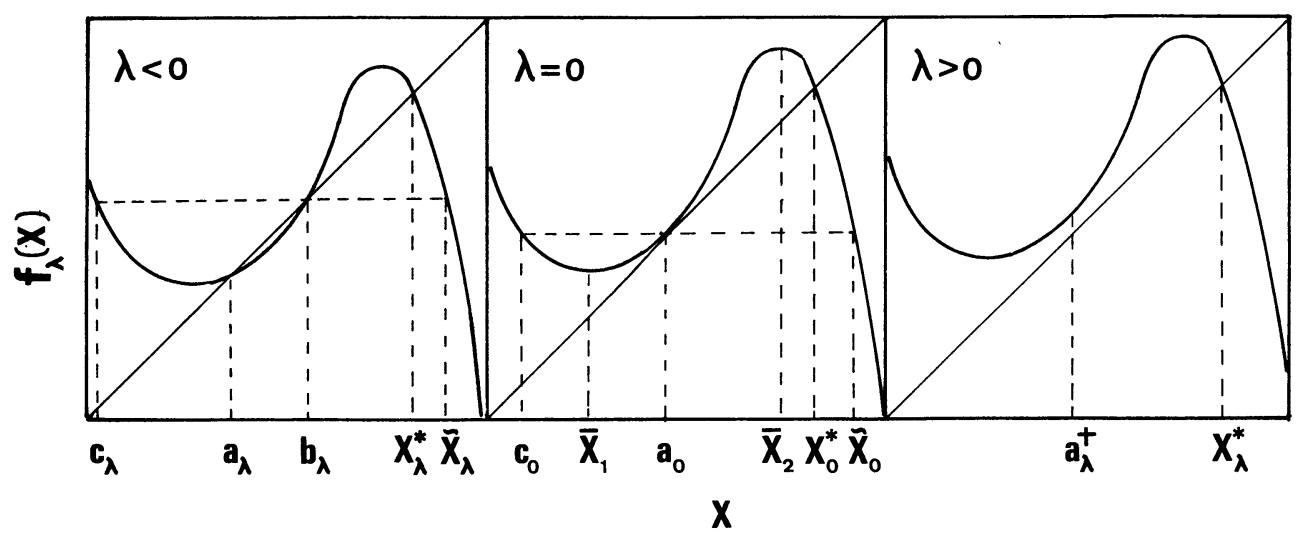

Fig. 1. - Graph of $f_{\lambda}(x)$. 


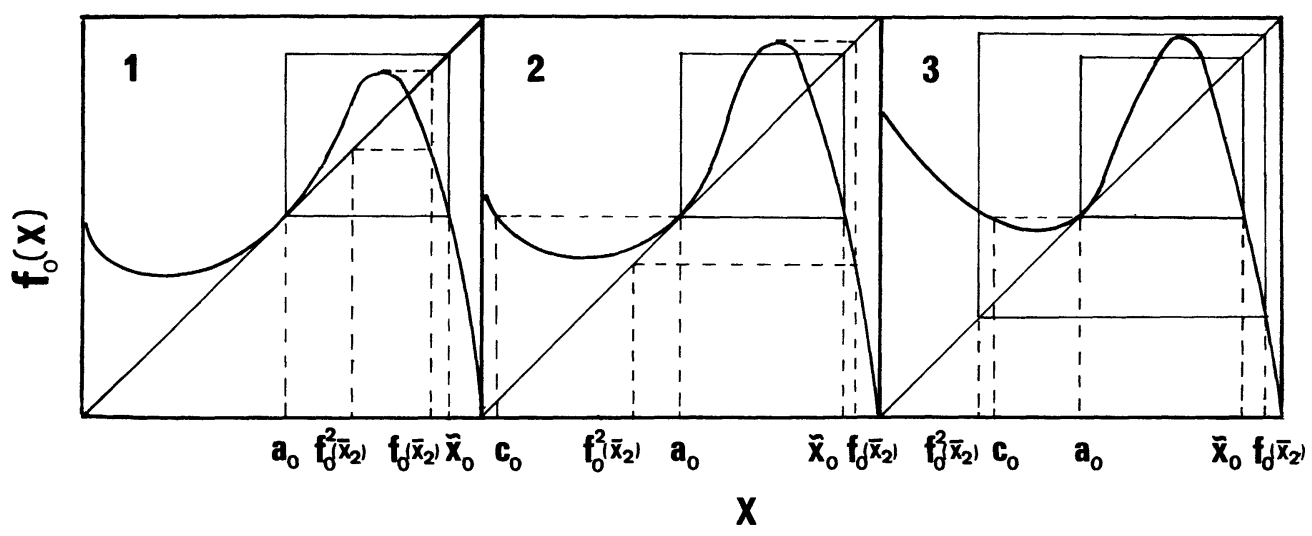

Fig. 3. - $f_{0}(x)$ corresponding to a discontinuous transition to chaos : 1) with complete hysteresis ;2) without hysteresis ; 3) with incomplete hysteresis.

is sufficient to imply that everything which goes out from $\left[a_{0}, \tilde{X}_{0}\right]$ is attracted by the fixed point $a_{0}$ for $\lambda=0$.

For $\lambda \gtrsim 0$, the measure (discrete or continuous) associated with the attractor $A_{\lambda}$ is mostly concentrated near the locus of the quasi fixed point $a_{\lambda}^{+}$(Fig. 1). Almost all orbits are recurrent in the neighbourhood of $a_{\lambda}^{+}$. The average number of successive points of these orbits in this neighbourhood diverges as $\lambda$ goes to zero. Thus we obtain an intermittency phenomenon which is analogous to the one described by Pomeau and Manneville in [14] (Fig. 4) except that in our case Kolmogorov-Sinaï entropy is not a continuous function of $\lambda$. This is due to the fact that $f_{\lambda}$ is homotopic to identity (in contrary to the models in [14]), so that the transition described here can be

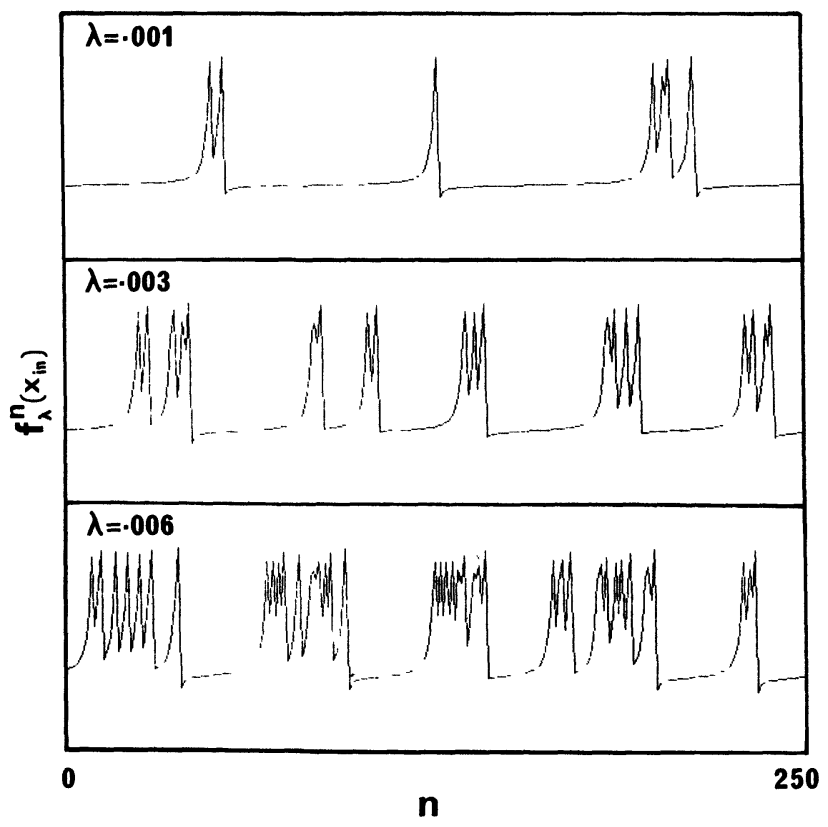

Fig. 4. - Typical intermittency patterns.

$$
f_{\lambda}(x)=-x^{3}+p x+2\left(\frac{p-1}{3}\right)^{3 / 2}+\lambda ; p=2.17
$$

successive iterates of an arbitrary initial point. considered as part of a transition from a system with a finite number of periodic orbits to a chaotic system. This is relevant for physical systems as e.g. some periodically driven oscillators [17]. It is remarkable that this intermittency gives a continuous character to a transition presupposed to be a discontinuous one.

For $\lambda \lesssim 0$, the fixed point $a_{\lambda}$ is stable, and it does trap $\mu$-almost all orbits issued from an initial condition in $\left[c_{\lambda}, \tilde{X}_{\lambda}\right]$; as a consequence hysteresis is impossible. For such values of $\lambda$, there remains in $\left[b_{\dot{\lambda}}, \tilde{X}_{\lambda}\right]$ an invariant Cantor set $K$ with zero Lebesgue measure, the dynamics on $K$ being homeomorphic to the full shift with two symbols.

Let us notice that for $\lambda<\lambda^{*}$ such that

$$
f_{i *}\left(\bar{X}_{2}\right)=\tilde{X}_{\text {i* }},
$$

there exists an invariant set $\left[b_{\lambda}, \tilde{X}_{\lambda}\right]$ which contains an attractor.

CASE 3. - Transition with incomplete hysteresis.

This corresponds to the conditions

$$
\begin{aligned}
& f_{0}\left(\bar{X}_{2}\right)>\tilde{X}_{0} \\
& f_{0}^{2}\left(\bar{X}_{2}\right)<c_{0} \\
& f_{0}^{3}\left(\bar{X}_{2}\right)<f_{0}\left(\bar{X}_{2}\right) .
\end{aligned}
$$

As in case 2, the first condition prevents the interval $\left[a_{0}, \widetilde{X}_{0}\right]$ from being invariant. By the second condition, some orbit issued from $\left[a_{0}, \tilde{X}_{0}\right]$ eventually reenters this interval. The last condition ensures invariance of the interval $\left[f_{0}^{2}\left(\bar{X}_{2}\right), f_{0}\left(\bar{X}_{2}\right)\right]$ (Fig. 3). With these conditions, we may have an invariant set partly in $\left[a_{0}, \tilde{X}_{0}\right]$ for $\lambda \lesssim 0$. Thus, in concurrence with orbits issued from $\left[a_{0}, \tilde{X}_{0}\right]$ and converging to the stable fixed point $a_{\lambda}$, we may find an attractor $A_{\lambda}$ at a finite distance from $a_{\lambda}$. The given conditions being verified in $C^{3}$ open sets, the same remarks hold for $\lambda$ slightly negative; this may allow hysteresis. The necessarily incomplete character of this hysteresis comes from the fact that when $\lambda$ decreases, the second inequality is obviously inverted before the first one; 
then the above-mentioned theorem applies and we end up as in case 2. The hysteresis breaks down when the metastable chaos, as described by Kaplan and Yorke [10, 18, 19], begins.

We disregard the case where the last condition is not satisfied since then the behaviour of orbits is intimately related to the nature at large distances of the function $f_{0}$.

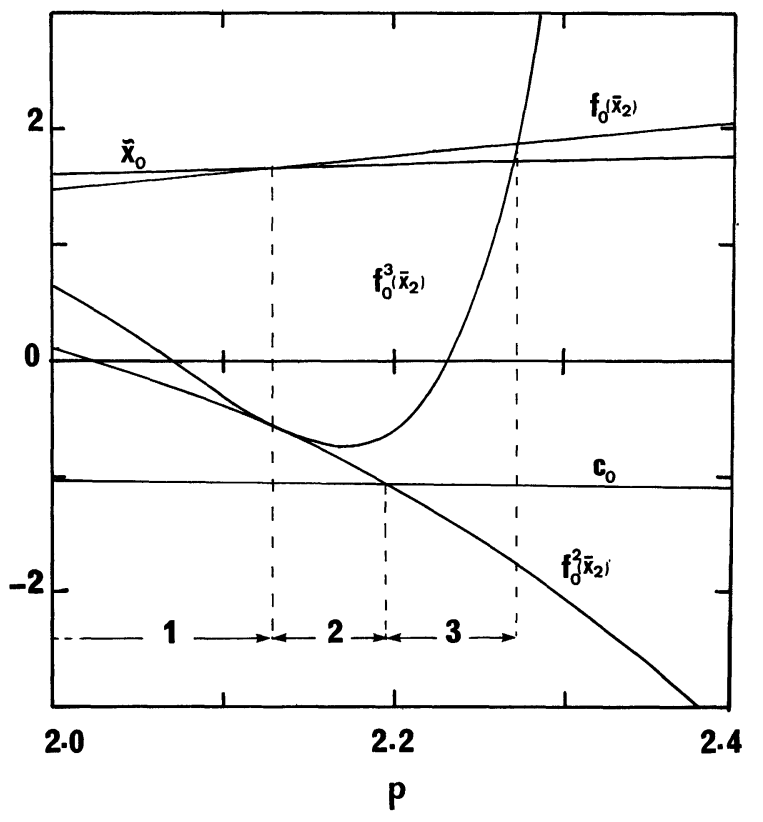

Fig. 5. - For $f_{0}(x)=-x^{3}+p x+2\left(\frac{p-1}{3}\right)^{3 / 2}$, this figure indicates the values of $p \geqslant 2$ such that the transition occurs : 1) with complete hysteresis ; 2) without hysteresis ; 3) with incomplete hysteresis. For $1 \leqslant p \leqslant 2$ we are always in case 1 .
These three cases can be realized by varying the parameter $p$ in the family (Fig. 5) :

$$
f_{\lambda, p}(x)=-x^{3}+p x+2\left(\frac{p-1}{3}\right)^{3 / 2}+\lambda ;
$$

this family is obviously $C^{3}$ with negative Schwarzian derivative and satisfies the conditions of the abovementioned theorem. We use this family to illustrate the intermittency phenomenon in figure 4.

Similar analysis can be done for other type of bifurcations. For example in the case of subcritical doubling-period bifurcation, the above-mentioned theorem yields conditions for incomplete hysteresis. However, for such a bifurcation, the intermittency (when it is observed) corresponds to another mechanism, namely the existence of homoclinic points in the invariant manifold of the fixed point.

In diffeomorphisms and flows of $\mathbb{R}^{n}$, similar phenomena can be exhibited e.g. in Lorenz model [1] where we observe a subcritical Hopf bifurcation with incomplete hysteresis $[10,18,19]$. Numerical studies on diffeomorphisms of $\mathbb{R}^{2}$ and flows of $\mathbb{R}^{3}$ will be reported in a forthcoming paper [13]. Let us point out that the three cases of discontinuous transitions we discussed in this letter can be observed in $\mathbb{R}^{2}$ diffeomorphisms defined by :

$$
(x, y) \rightarrow\left(f_{\lambda}(x)+y, b x\right)
$$

where $f_{\lambda}(x)$ is a real endomorphism which presents such transitions. This means that the classification also works for flows of $\mathbb{R}^{3}$, at least for those which are suspension of these diffeomorphisms.

\section{References}

[1] Lorenz, E. N., J. Atmos. Sci. 20 (1963) 130

[2] Ruelle, D., Takens, F., Commun. Math. Phys. 20 (1971) 167.

[3] Henon, M., Commun. Math. Phys. 50 (1976) 69.

[4] Coullet, P., Tresser, C., Arneodo, A., Phys. Lett. 72A (1979) 268.

[5] Feigenbaum, M. J., J. Stat. Phys. 19 (1978) 25.

[6] Tresser, C., Coullet, P., C.R. Hebd. Séan. Acad. Sci. Paris 287A (1978) 577.

[7] Derrida, B., Gervois, A., Pomeau, Y., J. Phys. A 12 (1979) 269.

[8] Collet, P., Eckmann, J. P., Lanford, O. E., Universal properties of maps of an interval, Preprint (1978).

[9] Arneodo, A., Coullet, P., Tresser, C., Phys. Lett. 70A (1979) 74.

[10] Afraľmovich, V. S., Bykov, V. V., ShIL'Nikov, L. P., Sov. Phys. Dokl. 22 (1977) 253.

KaPlan, J. L., Yorke, J. A., Commun. Math. Phys. 67 (1979) 93.
[11] Maurer, J., Libchaber, A., J. Physique Lett. 40 (1979) L-419.

[12] Gollub, J. P., Benson, S. V., Time-dependent instabilities and the transition to turbulent convection, Preprint submitted to $J$. Fluid Mech.

[13] Coullet, P., Tresser, C., Arneodo, A., First-order transition to stochasticity in some dynamical systems, in preparation.

[14] Pomeau, Y., Manneville, P., Intermittency : a generic phenomenon at the onset of turbulence. Talk given at the International Workshop on intrinsic stochasticity in plasma (Cargese 1979), to appear in Phys. Lett. A.

[15] Singer, D., S.I.A.M. J. Appl. Math. 35 (1978) 260.

[16] Misiurewicz, M., Absolutely continuous measures for certain maps of an interval, Preprint IHES/M/79/293 (1979).

[17] Afrămovich, V. S., Shil'nikov, L. P., Sov. Math. Dokl. 15 (1974) 206

[18] Pianigiani, G., Yorke, J. A., Trans. Am. Math. Soc. 252 (1979) 351 .

[19] Yorke, J. A., Yorke, E. D., J. Stat. Phys. 21 (1979) 263. 